\title{
A radio telescope in the Arctic region
}

\author{
A former ALMA prototype antenna has a new lease of life in the harsh conditions of Greenland, where it will play a \\ key role in very-long-baseline interferometry observations of supermassive black holes, explain Nimesh Patel and \\ Paul Ho.
}

T he Greenland Telescope (GLT) is a 12 -m diameter submillimetrewavelength radio telescope that was recently deployed at the Thule Air Force Base in Greenland (Fig. 1). The telescope provides the northernmost extended baseline of very-long-baseline interferometry (VLBI), providing a length of about 9,000 km from the Atacama Large Millimeter/submillimeter Array (ALMA) in Chile, and about the same length towards the west to the Submillimeter Array and the James Clerk Maxwell Telescope in Hawaii.

The main scientific goal of the GLT project is to carry out VLBI observations of supermassive black holes (SMBHs) in the nuclei of galaxies at 230,345 and 690 $\mathrm{GHz}$, achieving an angular resolution of tens of microarcseconds, as part of the Event Horizon Telescope (EHT). This will be sufficient to resolve the SMBH shadows, whose sizes are estimated to be five times larger than their event horizons ${ }^{1,2}$. The imaging of the SMBH shadows will directly measure the mass and spin, the only measurable quantities of SMBHs, and will be the first direct proof of the existence of SMBHs. The first target was the SMBH in M87 at a distance of 16 Mpc. In addition to the EHT, the GLT is also participating in the Global Millimeter VLBI Array observing campaigns to study similar active galactic nuclei sources, in order to understand accretion disks and jet physics ${ }^{3}$.

The GLT antenna also provides a unique opportunity to carry out various singledish submillimetre science projects in the 0.3-3 mm wavelength band for northern sources, from a very dry and stable site. The single-dish science applications include star formation, astrochemistry and largescale high-redshift galaxy surveys ${ }^{4}$. Various receivers will be fitted to support single-dish science, including heterodyne array receivers at 350 and $650 \mathrm{GHz}$, and $1.4 \mathrm{THz}$ hotelectron bolometer array receivers ${ }^{5}$.

The GLT project is a collaboration between the Smithsonian Astrophysical Observatory (SAO) and Academia Sinica Institute for Astronomy and Astrophysics (ASIAA), the two institutes involved in

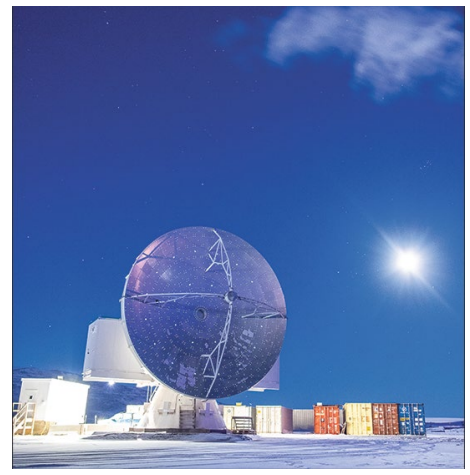

Fig. 1 | The Greenland Telescope, a Vertex dish that originally functioned as an ALMA prototype antenna.

the Submillimeter Array on Mauna Kea, Hawaii. The GLT antenna was originally the 12-m ALMA North America prototype antenna, which was located near Socorro, New Mexico ${ }^{6}$. In 2011, the National Science Foundation and Associated Universities, Inc. (AUI)/NRAO awarded the antenna to the SAO, in a proposal from Paul Ho, representing the ASIAA/SAO team, for relocation to Greenland.

ASIAA has led the effort on the GLT project, raising most of the budget for the refurbishment of the antenna and leading the successful deployment in Thule. In the period 2012-2016, ASIAA and the SAO team worked at a rapid pace to refurbish the antenna with several new components to meet the operating specifications of extreme Arctic conditions $\left(-30^{\circ} \mathrm{C}\right.$ in Thule; $-60^{\circ} \mathrm{C}$ at the Summit station). The new components include parts of the backup structure, a set of carbon-fibre quadruped legs, a sub-reflector and its hexapod controller, a servo system including motors, gearboxes and an antenna control unit, and a set of elevation bearings ${ }^{7}$.

The GLT currently has three VLBI receivers, operating in the 84-96, 213-243 and $271-377 \mathrm{GHz}$ bands ${ }^{8}$. The receivers and all backend systems were tested in autumn 2017 and met their performance specifications. It has been very helpful to have a built-in set of calibration and diagnostic systems in all of the instrumentation ${ }^{9}$. Single-dish testing activities began in December 2017. The first light GLT observations of the Moon in continuum mode were obtained in December 2017, at 86 and $230 \mathrm{GHz}$. Then followed spectral line observation of the $\mathrm{CO} J=2-1$ line from Orion $\mathrm{BN} / \mathrm{KL}^{10}$. First VLBI fringes were obtained in January 2018. Successful EHT observations were then carried out in April $2018^{10}$.

Currently, system performance is being improved in preparation for the 2019 observing season. Preliminary aperture efficiency measurements showed low values of about 0.5 at $86 \mathrm{GHz}$ and 0.25 at $230 \mathrm{GHz}$, which are attributed to the alignment of the surface of the telescope. Photogrammetry and holographic surface measurements and adjustments are now in progress at Thule, as of September 2018. Commissioning of the $345 \mathrm{GHz}$ operation, and a test EHT observing run, took place in October 2018.

The future plan is to move the antenna to a site near the Summit station in Greenland, at an altitude of 3,100 m above sea level, where the atmospheric opacity is far lower and more stable, allowing superb transparency at frequencies higher than $345 \mathrm{GHz}$ (ref. ${ }^{11}$ ). This plan is still at a preliminary stage. It will require substantial development of new infrastructure near the Summit station and additional funding support over a span of three years.

Nimesh A. Patel ${ }^{1 \star}$ and Paul T.P. Ho ${ }^{2}$

${ }^{1}$ Harvard-Smithsonian Center for Astrophysics, Harvard University, Cambridge, MA, USA.

${ }^{2}$ Academia Sinica Institute of Astronomy and Astrophysics, Taipei, Taiwan.

*e-mail:npatel@cfa.harvard.edu

Published online: 3 December 2018 https://doi.org/10.1038/s41550-018-0649-z

\footnotetext{
References

1. Doeleman, S. S. Nat. Astron. 1, 646 (2017)

2. Psaltis, D. \& Özel, F. Phys. Today 71, 70-71 (April, 2018).

3. Inoue, M. et al. Radio Sci. 49, 564-571 (2014).

4. Hirashita, H. et al. Publ. Astron. Soc. Japan 68, 1 (2016).

5. Grimes, P. K. et al. Proc. SPIE 9153, $91531 \mathrm{~V}$ (2014).

6. Mangum, J. G. et al. Publ. Astron. Soc. Pac. 118, 1257-1301 (2006).

7. Raffin, P. et al. Proc. SPIE 9906, 99060U (2016).

8. Chen, M.-T. et al. Proc. SPIE 10700, $107000 \mathrm{H}$ (2018).

9. Kubo, D. et al. Proc. SPIE 10708, 1070816 (2018).

10. Matsushita, S. et al. Proc. SPIE 10700, 1070029 (2018).

11. Matsushita, S. et al. Publ. Astron. Soc. Pac. 129, 025001 (2016).
} 